\title{
Computer-aided design/ computer-aided manufacturing crown survival rates
}

\author{
Abstracted from \\ Encke BS, Heydecke G, Wolkewitz M, Strub JR. \\ Results of a prospective randomised controlled trial of posterior $\mathrm{ZrSiO}_{4}$-ceramic crowns. \\ J Oral Rehabil 2009; 36: 226-235 \\ Address for correspondence: Dr Birgit Encke, Department of Prosthodontics, \\ School of Dentistry, University Medical Centre, Hugstetterstrasse 55, \\ D-79106 Freiburg im Breisgau, Germany. E-mail: birgit.encke@uniklinik-freiburg.de
}

\section{Question: Do ceramic computer-aided design/ computer-aided manufacturing crowns have similar long-term outcomes to conventional gold crowns?}

Design A randomised controlled trial (RCT) was conducted in a dental school.

Intervention A total of 224 patients were randomised into two treatment groups, with 123 patients having teeth restored with KaVo Everest high-performance ceramic (HPC) crowns, fabricated by a computer-aided manufacturing procedure (KaVo Dental $\mathrm{GmbH}$, Biberach an der Riss, Germany) and 101 patients receiving gold crowns. All crowns were conventionally cemented with glass-ionomer cement. Outcome measure Crowns were assessed for loss of vitality, surface roughness, fractures, marginal integrity, secondary caries at the crown margin, margin discolouration, marginal gap and crown loss at set time intervals of 6 and 12 months. The cumulative incidence of clinical complications was used as the criterion for failure. Time-to-event (failure) analysis was used for survival data. The incidence-free survival probabilities were then assessed using Kaplan-Meier analysis. A proportional hazard model (Cox) was used to test the treatment effect. Results After an observation period of 6,12 and 24 months, the prospective survival rates (Kaplan-Meier) for the KaVo Everest HPC crowns were $97.9 \%, 95.1 \%$ and $89.8 \%$, and for the gold crowns were $100 \%, 94.8 \%$ and $92.7 \%$, respectively. There were no significant differences between the two groups (P 0.2). The 1-year failure rates were $4.9 \%$ for the KaVo Everest HPC crowns and 5.2\% for the gold crowns. The 1-year cumulative risks for loss of vitality, secondary caries, fractures, loss of crown and extraction of abutment of the analysed abutments (88) were $8.9 \%, 0 \%, 0 \%, 1.1 \%$ and $1.1 \%$, respectively, for the gold crowns and $2.8 \%, 0 \%, 4.7 \%, 0 \%$ and $0.9 \%$, respectively, for the ceramic crowns (107 analysed abutments). No perfect marginal fit was shown by $49.5 \%$ of the evaluated ceramic crowns and $26.1 \%$ of the gold crowns.

Conclusions The 12-month results indicate that Everest HPC crowns are suitable for posterior restorations, provided that an adequate tooth reduction is possible. The marginal fit shows potential for improvement.

\section{Commentary}

There is a need for trials that compare different types of prostheses, and this study represents a small but growing number of RCT that compare one type of crown with another. In the results, the authors state that there were no significant differences between groups with respect to prosthesis survival.

Study strengths in this trial included a participant flow diagram, adequate randomisation and the fact that both groups appeared to be similar at baseline for both age and gender. There were differences between the groups at baseline for tooth vitality, however, with $67.7 \%$ vitality in the control group but only $57.7 \%$ in the test group. This difference could be relevant as there is more tooth reduction with teeth prepared for Everest HPC crowns.

Internal validity refers to how well a study was conducted, whereas external validity refers to the ability to extrapolate findings to one's clinical practice, and there were some shortcomings with this trial in both domains. A common outcome in fixed prosthodonics is time to failure, which typically takes many years. The most glaring weakness with this study is that only a 12-month observation period was completed at publication. Extrapolating to one's clinical practice is difficult here, as prosthesis survival studies typically run from 5-20 years to allow for failures to occur. This study ultimately plans to follow patients for 60 months.

There are several types of bias in RCT. Selection bias, which refers to the method of randomisation, was minimised in this trial with both generation of sequences and allocation concealment prior to assignment. Central randomisation, which is perhaps the best way to ensure proper allocation concealment, ${ }^{1}$ was done using a Bernoulli distribution (a statistical method where only two outcomes are equally possible).

Performance bias appears to have been avoided in that both groups were treated equally, apart from the intervention itself. It was unclear, however, if the required restorative and periodontal care was provided prior to or after randomisation. Detection bias, the inability to blind or mask investigators and participants, is difficult to avoid in trials such as these where both groups can clearly tell to which arm of the trial they have been assigned. It is almost always possible to blind outcomes-assessors, though, and there was no mention of this in the study. This is relevant as non-blinded investigators assessing outcomes can bias trial results.

Attrition bias refers to the dropout rate, and whether attrition rates were similar in both groups. The dropout rate was about $13 \%$ for both groups, well within an acceptable range. Attrition rates above $20 \%$ can affect the validity of a trial. There was no mention 


\section{RESTORATIVE DENTISTRY}

of an intention-to-treat (ITT) analysis, which has two components: including all dropouts into the data analysis, and keeping the dropouts assigned to their original groups. ITT is important as it is an incorrect assumption that dropouts will be equal in both groups, and these inequalities can again lead to biased results. This trial is planned to run for up to 60 months, which would be considered the minimum amount of time for trials of this type. Given a 13\% dropout rate at 12 months, the attrition rate at 60 months could seriously affect the validity of the study, even with an ITT analysis. ${ }^{2}$

Another important factor in trial quality is sample size calculation. This relates to the ability of a study to find a difference between groups if one actually exists, in an effort to avoid a type II error (falsely accepting the null hypothesis). Typically, a power calculation is done to determine an appropriate sample size. Trials are often underpowered, increasing the chance of a type II error, but artificially increasing the size of a trial can lead to statistically significant differences between groups which may be clinically irrelevant. There was no mention of a power calculation in this trial, and the fact that there were no significant differences between groups means that either there truly was no difference, the observation period was too short, or the study did not have enough power to detect a difference. Another reason for a power calculation is to ensure that enough participants remain in the trial at completion which, again, may be a concern at 60 months.

A trial should limit the number of outcomes to one or two primary outcomes. In this trial, there were several outcomes, some of which were surrogate outcomes, such as marginal integrity or surface roughness. The addition of outcomes increases the likelihood of finding statistically significant results. Crowns were considered failures if there were partial or complete crown fractures, secondary caries, crown loss, or tooth extraction. Curiously, the definition of failure included loss of vitality, and since there were more gold crowns that lost vitality, excluding this outcome would have pushed survival rates toward statistical significance.

Failure rates were given for the 6-, 12- and 24-month examination periods. The main result was expressed as a hazard ratio, the ratio of failure of the test group divided by the control group. This result was expressed as 1.79 (95\% confidence interval, $0.74-4.32 ; P 0.2$ ). This means that the Everest HPC group had a $79 \%$ greater failure rate than the control group. Although the hazard ratio is statistically insignificant, with a $P$-value greater than 0.05 , the confidence interval merits further exploration. The $95 \%$ confidence interval incorporates the null value of " 1 ", translating to a $P$-value greater than 0.05 , but the confidence interval is skewed well toward higher rates of failure for Everest HPC crowns. ${ }^{3}$ This implies that, whereas failure rates for Everest HPC crowns may be $26 \%$ lower than for gold crowns, they may be more than fourfold higher than full gold crowns. Again, with no sample size calculation, it is possible the study is under-powered to detect a statistical difference. Additionally, as a 5-year examination period is planned, it is possible that the hazard ratio at that point might reach statistical significance, assuming the attrition rate does not greatly increase.

This article illustrates one of the major difficulties when conducting RCT in restorative dentistry: a common outcome is time to failure, which may take many years, allowing for high attrition rates which then have a negative affect on study validity. In summary, this trial, at moderate risk for bias, found no statistically significant differences between gold and Everest HPC crowns for a 12-month observation period. A longer observation period, with a sample size calculation and low attrition rates is needed to establish clinically relevant findings.

Elliot Abt

Department of Dentistry, Illinois Masonic Medical Center, Chicago, Illinois, USA

1. Higgins JPT, Green S (editors). Cochrane Handbook of Systematic Reviews for Interventions. Version 5.0.1. The Cochrane Collaboration 2008; section 8.10.2.

2. Montrori VM, Guyatt GH. Intention-to-treat principle. Can Med Assoc / 2001; 165: 1339-1341.

3. Greenberg BL, Kantor ML. The clinician's guide to the literature. I Am Dent Assoc 2009; 140: 48-54.

Evidence-Based Dentistry (2010) 11, 25-26. doi:10.1038/sj.ebd.6400709 